University of Wollongong

Research Online

Australian Institute for Innovative Materials -

Papers

Australian Institute for Innovative Materials

$1-1-2013$

The flux pinning mechanism, and electrical and magnetic anisotropy in Fe1.04Te0.6Se 0.4 superconducting single crystal

Mahboobeh Shahbazi-Manshadi

University of Wollongong, msm979@uowmail.edu.au

Xiaolin Wang

University of Wollongong, xiaolin@uow.edu.au

SX. Dou

University of Wollongong, shi@uow.edu.au

H Fang

University of Wollongong, fh640@uowmail.edu.au

C T. Lin

Max-Planck-Institut fur Festkorperforschung, Stuttgart

Follow this and additional works at: https://ro.uow.edu.au/aiimpapers

Part of the Engineering Commons, and the Physical Sciences and Mathematics Commons

Research Online is the open access institutional repository for the University of Wollongong. For further information contact the UOW Library: research-pubs@uow.edu.au 


\title{
The flux pinning mechanism, and electrical and magnetic anisotropy in Fe1.04Te0.6Se 0.4 superconducting single crystal
}

\author{
Abstract \\ The temperature and magnetic field dependences of the magnetization and critical current density of \\ Fe1.04Te0.6Se0.4 single crystals have been investigated, and the flux pinning mechanism has been \\ analysed. The normalized pinning force (fp Fp/F p,max) vs. $h$ (H/Hirr) curves, are scaled using the Dew- \\ Hughes theory, $f(h) \approx h p(1-h) q$ with $p 1.35$ and $q 3.06$. The angular dependence of the resistivity under \\ different magnetic fields shows a dip-like structure, below the superconducting transition temperature. \\ The anisotropic value of 2 was obtained using Ginzburg-Landau theory.

\section{Keywords} \\ single, superconducting, 4, 6se0, 04te0, crystal, fe1, flux, anisotropy, magnetic, electrical, mechanism, \\ pinning

\section{Disciplines} \\ Engineering | Physical Sciences and Mathematics \\ Publication Details \\ Shahbazi-Manshadi, M., Wang, X., Dou, S. X., Fang, H. and Lin, C. T. (2013). The flux pinning mechanism, \\ and electrical and magnetic anisotropy in Fe1.04Te0.6Se0.4 superconducting single crystal. In \\ Proceedings of the 12th Joint MMM-Intermag Conference: Fundamental Properties and Cooperative \\ Phenomena, 14-18 January, Chicago, Illinois, USA. Journal of Applied Physics, 113 17E115-1-17E115-3.
}




\section{AIP $\mid \begin{aligned} & \text { Jumanal } \\ & \text { Applied Physics }\end{aligned}$}

The flux pinning mechanism, and electrical and magnetic anisotropy in Fe1.04Te0.6Se 0.4 superconducting single crystal

M. Shahbazi, X. L. Wang, S. X. Dou, H. Fang, and C. T. Lin

Citation: J. Appl. Phys. 113, 17E115 (2013); doi: 10.1063/1.4794134

View online: http://dx.doi.org/10.1063/1.4794134

View Table of Contents: http://jap.aip.org/resource/1/JAPIAU/v113/i17

Published by the AIP Publishing LLC.

Additional information on J. Appl. Phys.

Journal Homepage: http://jap.aip.org/

Journal Information: http://jap.aip.org/about/about_the_journal

Top downloads: http://jap.aip.org/features/most_downloaded

Information for Authors: http://jap.aip.org/authors

\section{ADVERTISEMENT}

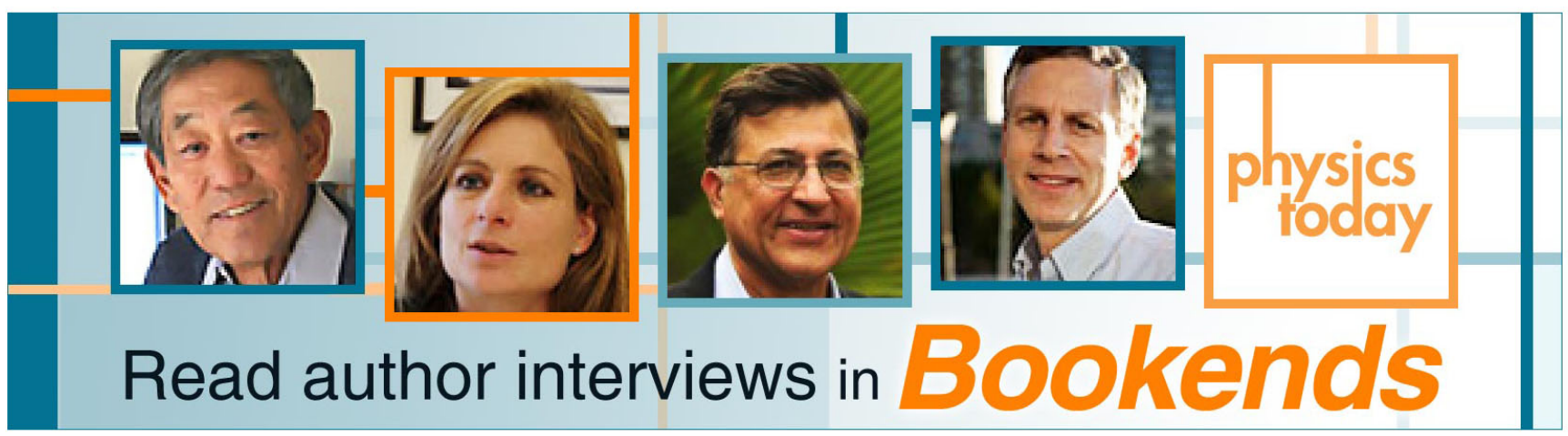




\title{
The flux pinning mechanism, and electrical and magnetic anisotropy in $\mathrm{Fe}_{1.04} \mathrm{Te}_{0.6} \mathrm{Se}_{0.4}$ superconducting single crystal
}

\author{
M. Shahbazi, ${ }^{1, a)}$ X. L. Wang, ${ }^{1}$ S. X. Dou, ${ }^{1}$ H. Fang, ${ }^{1}$ and C. T. Lin $^{2}$ \\ ${ }^{1}$ Institute for Superconducting and Electronic Materials, University of Wollongong, Australia \\ ${ }^{2}$ Max-Planck-Institut für Festkörperforschung, Heisenbergstr. 1, 70569 Stuttgart, Germany
}

(Presented 15 January 2013; received 30 October 2012; accepted 27 November 2012; published online 4 March 2013)

\begin{abstract}
The temperature and magnetic field dependences of the magnetization and critical current density of $\mathrm{Fe}_{1.04} \mathrm{Te}_{0.6} \mathrm{Se}_{0.4}$ single crystals have been investigated, and the flux pinning mechanism has been analysed. The normalized pinning force $\left(f_{\mathrm{p}}=F_{\mathrm{p}} / F_{\mathrm{p}, \max }\right)$ vs. $h\left(H / H_{\text {irr }}\right)$ curves, are scaled using the Dew-Hughes' theory, $f(h) \approx h^{\mathrm{p}}(1-h)^{\mathrm{q}}$ with $p=1.35$ and $q=3.06$. The angular dependence of the resistivity under different magnetic fields shows a dip-like structure, below the superconducting transition temperature. The anisotropic value of 2 was obtained using GinzburgLandau theory. (C) 2013 American Institute of Physics. [http://dx.doi.org/10.1063/1.4794134]
\end{abstract}

Following the discovery of superconductivity in the $R E F e P n \mathrm{O}(R E=$ rare earth, $P n=\mathrm{P}$ or As $),{ }^{1-5}$ doped $A \mathrm{Fe}_{2} \mathrm{As}_{2}$ $\left(A=\right.$ alkaline or alkaline earth metal) $,{ }^{6,7} \mathrm{LiFeAs},{ }^{8}$ and $\left(\left(\mathrm{Sr}_{4} M_{2} \mathrm{O}_{6}\right) \mathrm{Fe}_{2} P n_{2}\right)(M=\mathrm{Sc} \text {, Ti, or } \mathrm{V})^{9,10}$ families of iron pnictide superconductors, the observation of superconductivity in tetragonal FeSe (Ref. 11) has opened up a new window of opportunity to further understand the mechanism of superconductivity in the iron pnictides. The iron chalcogenide FeSe compounds are of great interest from the viewpoints of both the vortex properties in the mixed state and practical application. This is largely due to the relatively simple structure and similarity in the Fermi surface $\left(\mathrm{E}_{\mathrm{F}}\right)$ of these arsenic-free compounds to the other pnictide superconductors. The $\mathrm{E}_{\mathrm{F}}$ surfaces of $\mathrm{FeSe}$ and $\mathrm{FeTe}$ contain cylindrical hole and electron sections at the centre and the corner of the Brillouin zone, respectively. ${ }^{12}$ It has proven difficult, however, to grow homogeneous superconductive single crystals. Therefore, attention has been paid to some extent to the Te doped systems. ${ }^{13}$ $\mathrm{FeSe}_{1-x} \mathrm{Te}_{x}$ compounds have the tetragonal structure, where the $\mathrm{Fe}(\mathrm{Se} / \mathrm{Te})$ layers stack along the $c$-axis, and have $T_{\mathrm{c}}$, as high as $15 \mathrm{~K}^{13-15}$ The antiferromagnetic order of FeTe is gradually suppressed by increasing $x$ in $\mathrm{FeTe}_{1-x} \mathrm{Se}_{x}$, and the maximum $T_{\mathrm{c}}$ is achieved for $x=0.5{ }^{16}$

It is crucial to understand the pinning mechanism from both the practical and the fundamental point of view. There are two main interactions between vortices and pinning centres in type II superconductors: the magnetic interaction and the core interaction. ${ }^{17}$ The magnetic interaction is due to the interaction at interfaces between superconducting and non-superconducting materials parallel to the applied magnetic field. The core interaction covers pinning due to the variation in the transition temperature $\left(\delta T_{c}\right)$ and pinning because of the variation in the charge carrier mean free path near lattice defects $(\delta l) .{ }^{17}$ For the $\mathrm{FeTe}_{0.5} \mathrm{Se}_{0.5}$ single crystal, it has been found that the dominant pinning mechanism is $\delta l$ pinning, which is related to small bundle vortex pinning due to randomly distributed weak pinning centres. ${ }^{18}$ Yadav et al.

\footnotetext{
a) Author to whom correspondence should be addressed. Electronic mail: msm979@uow.edu.au.
}

have studied the flux pinning force, $F_{\mathrm{p}}$, in $\mathrm{FeTe}_{0.6} \mathrm{Se}_{0.4}$ and found that the obtained $h_{\max }=0.28$ (where $h_{\max }$ is the field corresponding to the maximum pinning force density, normalized with respect to the irreversibility field $H_{\text {irr }}$ ), which can be understood in terms of $\delta l$ pinning with a mixture of surface pinning and the point core pinning due to normal pinning centres, with different ranges of the pinning interactions. ${ }^{19}$ In this work, the vortex pinning mechanisms, pinning potential, and anisotropy of $\mathrm{Fe}_{1.04} \mathrm{Te}_{0.6} \mathrm{Se}_{0.4}$ single crystal have been studied systematically by magnetic and transport measurements at different temperatures.

Single crystals of $\mathrm{Fe}_{1.04} \mathrm{Te}_{0.6} \mathrm{Se}_{0.4}$ were prepared by a self-flux method. Details of the single crystal growth are reported elsewhere. ${ }^{20}$ The as-grown crystals were cleaved and cut into a rectangular shape for magnetic and transport measurements using a physical properties measurement system (PPMS, Quantum Design). The angular dependence of the resistivity was also measured with the angle, $\theta$, ranging from $0^{\circ}$ to $180^{\circ}$, where $\theta=0^{\circ}$ corresponds to $H / / c$ and $\theta=90^{\circ}$ to $H / / a b$, respectively.

Figure 1(a) shows typical hysteresis $(M-H)$ loops collected at several temperatures below $T_{\mathrm{c}}$. The minimum in the magnetic moment located at nearly zero field represents the first magnetization peak. The field completely penetrates into the bulk of the sample after zero field cooling. ${ }^{18}$ The second magnetization peak (SMP) can be seen at $4 \mathrm{~K}, 7 \mathrm{~K}$, and $8 \mathrm{~K}$. The onset $\left(H_{S M P}^{\text {onset }}\right)$ and peak $\left(H_{S M P}^{\text {peak }}\right)$ positions of the SMP are indicated by arrows in Fig. 1 (a) for $\mathrm{T}=4 \mathrm{~K}$. Both the $H_{S M P}^{\text {onset }}$ and the $H_{S M P}^{\text {peak }}$ positions move to lower magnetic fields as the temperature increases from 4 to $10 \mathrm{~K}$ and completely disappear at $\mathrm{T}=11 \mathrm{~K}$.

$J_{\mathrm{c}}$ was calculated at various temperatures from these $M-H$ loops by using the Bean model. ${ }^{21}$ For a rectangular shaped crystal with dimensions $c<a<b$, when $H / / c$, the $J_{\mathrm{c}}(H)$ is given by

$$
J_{\mathrm{c}}(H)=20 * \Delta m(H) /(a(1-a / 3 b)),
$$

where $\Delta m$ is the difference between the magnetizations measured during the return and the forward legs of the $M-H$ loops 

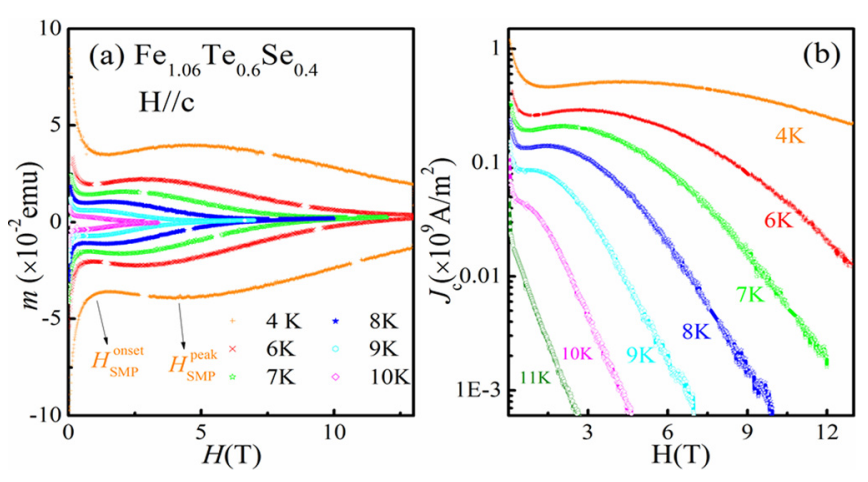

FIG. 1. (a) M-H loops of $\mathrm{BaFe}_{1.04} \mathrm{Te}_{0.6} \mathrm{Se}_{0.4}$ single crystal (b) and $J_{\mathrm{c}}$ vs. field at several temperatures.

for $H / / c$ and $a$ and $b$ are the length and width of the sample $J_{\mathrm{c}}(H)$ (Fig. 1(b)) shows a fast decrease at low fields $(H<0.5 \mathrm{~T})$, followed by weak field dependence at high fields at $T=4 \mathrm{~K}$. The in-field $J_{\mathrm{c}}$ is as high as $1.2 \times 10^{9} \mathrm{~A} / \mathrm{m}^{2}$ at $4 \mathrm{~K}$ and zero magnetic field. This value is slightly higher than the reported value $\left(1 \times 10^{9} \mathrm{~A} / \mathrm{m}^{2}\right.$ at $T=1.8 \mathrm{~K}$ and low field $)$ in a $\mathrm{FeTe}_{0.6} \mathrm{Se}_{0.4}$ single crystal. ${ }^{19,22}$ It is likely that this is due to the enhanced iron concentration in $\mathrm{Fe}_{1.04} \mathrm{Te}_{0.6} \mathrm{Te}_{0.4}$ single crystal, which introduces more defects into the crystal structure, and consequently, higher pinning potential into the system.

In order to understand the flux pinning mechanism which controls the vortex pinning force, it is useful to look at the variation of pinning force density with the magnetic field. In the mixed state of type II superconductors, the pinning force should follow the same general relationship, if the flux pinning is dominated by a single mechanism. ${ }^{23}$ The pinning force, defined as $F_{\mathrm{p}}=\mu_{0} H \times J_{c}$, can be calculated from the $J_{\text {c }}$ values shown in Fig. 1(b). In Fig. 2, we plot the reduced pinning force $f$ versus the reduced magnetic field $h\left(f=F_{\mathrm{p}} /\right.$ $\left.F_{\text {p,max }}, h=H / H_{\text {irr }}\right)$ at the same temperature as in Fig. 1(b). There are various methods to determine the irreversibility field, $H_{\text {irr }}$, from the magnetization and resistivity measurements. ${ }^{24}$ Here, we use $H_{\text {irr }}$ as the field at which $J_{\mathrm{c}}(H)$ is extrapolated to $10^{6} \mathrm{~A} / \mathrm{m}^{2}$. The curves show a scaling behaviour at $T<10 \mathrm{~K}$, indicating that a single pinning mechanism dominates at this temperature range. We fit these data within the Dew-Hughes scenario

$$
F_{p} \approx h^{p}(1-h)^{q} .
$$

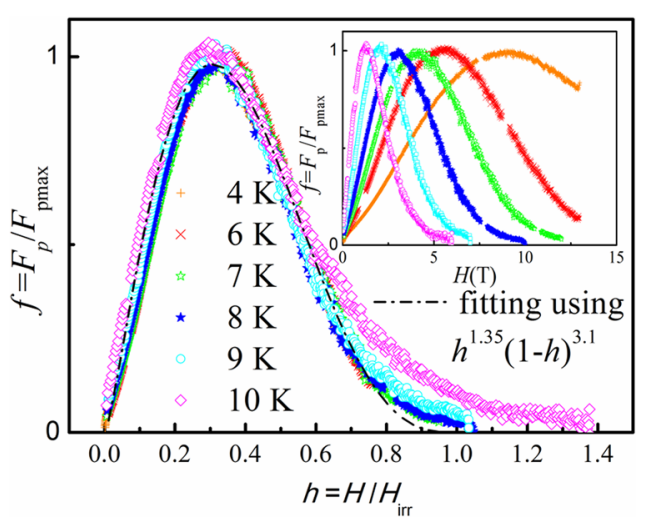

FIG. 2. Field dependence of the reduced pinning force, with the fitting results obtained from $h^{p}(1-h)^{q}$. Inset shows $F_{\mathrm{p}} / F_{\mathrm{p}, \max }$ as function of field.
The best fit of the curves (dashed-dotted line in Fig. 2) is obtained with an $f(h)$ dependence given by $h^{1.35}(1-h)^{3.1}$. The obtained $p$ and $q$ values are slightly lower than the reported values of $p=1.54$ and $q=3.8$ in $\mathrm{FeTe}_{0.6} \mathrm{Se}_{0.4}$ single crystal, ${ }^{19}$ possibly due to excess iron concentration in this compound. The value of $p /(p+q) \approx 0.3$ agrees well with the peak positions of these compounds in the $f$ versus $h$ plot, and it is in good agreement with the reported value of $h_{\max } \approx 0.28$ for $\mathrm{FeTe}_{0.6} \mathrm{Se}_{0.4}$ single crystal. ${ }^{19}$ It should be noted that according to the Dew-Hughes theory, ${ }^{23}$ point pinning is expected to lead to $p=1$ and $q=2$, with $F_{\mathrm{p} \text {,max }}$ occurring at $h_{\text {max }} \approx 0.33$, whereas in a system dominated by grain boundary pinning, $h_{\max } \approx 0.2$. In the case of pinning due to variation in the superconducting order parameter, however, $h_{\max } \approx 0.7$. In our case, $h_{\max } \approx 0.3$, implying $\delta l$ pinning with a mixture of point pinning and grain boundary pinning.

The temperature dependence of the resistivity for $H / / c$, is shown in Fig. 3(a), where the onset of $T_{\mathrm{c}}$ gradually shifts to lower temperatures with increasing magnetic field. It is worth noting that the shape of $\rho_{\mathrm{ab}}(T)$ with $H / / c$ is comparable to those for $(\mathrm{Ba}, \mathrm{K}) \mathrm{Fe}_{2} \mathrm{As}_{2}$ and $(\mathrm{Ba}, \mathrm{Rb}) \mathrm{Fe}_{2} \mathrm{As}_{2}$ single crystals, ${ }^{25,26}$ and it is different from the shapes for $\mathrm{SmFeAsO}_{0.8} \mathrm{~F}_{0.2}$ and $\mathrm{NdFeAsF}_{0.82} \mathrm{~F}_{0.18}$ single crystals, ${ }^{27,28}$ where resistive tails were clearly observed for $\mathrm{H} / / c$. According to the thermally activated flux flow model, the resistivity can be described by the following Arrhenius equation:

$$
\ln R(T, H)=\ln \mathrm{R}_{0}-U_{\mathrm{o}} / T .
$$

Therefore, the activation energy, $U_{\mathrm{o}}(H)$, is the slope of the lower part of the curve in the Arrhenius plot. In order to study the flux motion in $\mathrm{Fe}_{1.06} \mathrm{Te}_{0.6} \mathrm{Te}_{0.4}$ single crystal, we plot the resistivity as a function of $1 / T$ at different magnetic fields up to $13 \mathrm{~T}$. Figure 3(b) shows the Arrhenius plot of the resistivity for $H / / c$. The linear dependence of $\operatorname{Ln} \rho$ vs. $1 / T$ in the lower part of the curves indicates that this part can be described by the thermally activated flux flow model. ${ }^{29}$ Similar measurements were performed for $\mathrm{H} / / a b$ and also for several angles, from $0^{\circ}$ up to $90^{\circ}$ for $H=6 \mathrm{~T}$, and $U_{\mathrm{o}}(H)$ was calculated from the corresponding data.

The magnetic field dependence of the pinning potential for $\mathrm{H} / / c$ and $\mathrm{H} / / a b$ is shown in Fig. 4(a). The best fit to the experimental data yields a value of the pinning potential of $404 \mathrm{~K}$ for $H / / a b$ at $H=0.1 \mathrm{~T}$. The activation energy drops very slowly with increasing applied magnetic field for $H<5 \mathrm{~T}$, scaled as $H^{-0.1}$, and then decreases slowly as $H^{-0.61}$

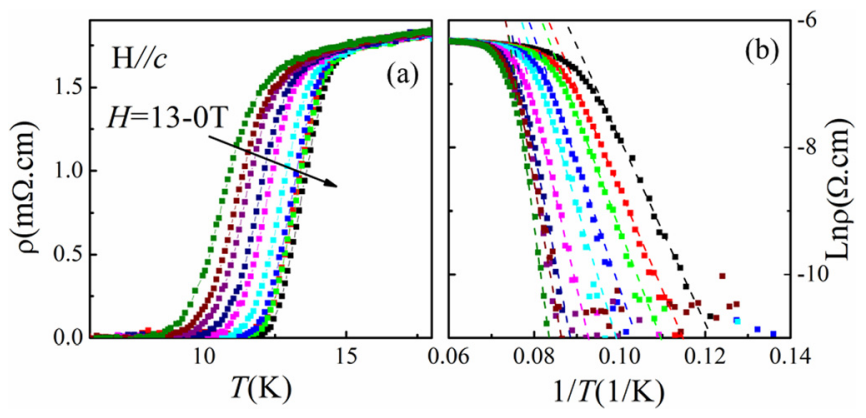

FIG. 3. (a) Temperature dependence of resistivity under different magnetic fields for $H / / c$. (b) Arrhenius plots of the resistivity for the same magnetic fields. 


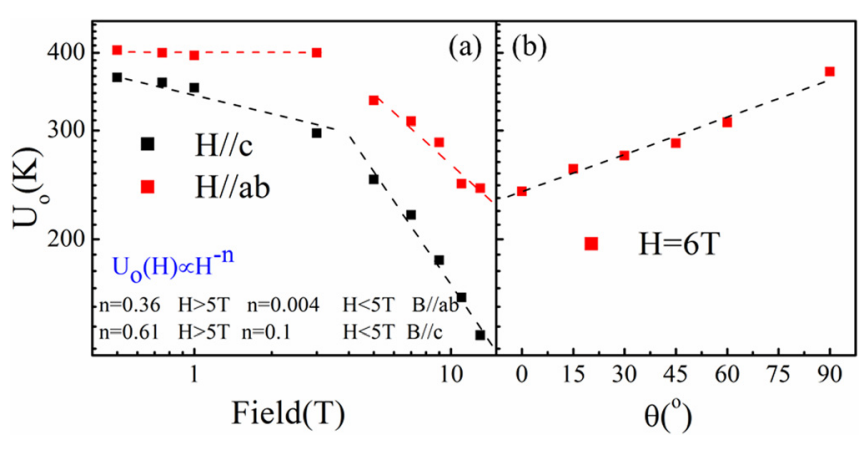

FIG. 4. (a) Magnetic field dependence of $U_{\mathrm{o}}$ for $H / / a b$ and $H / / c$. (b) Angular dependence of $U_{\mathrm{o}}$ for $H=6 \mathrm{~T}$.

for $H>5 \mathrm{~T}$. This indicates that the pinning potential is almost field independent for $H<5 \mathrm{~T}$. The angular dependence of the pinning potential for $H=6 \mathrm{~T}$ is shown in Fig. 4(b). The pinning potential values increase when the sample is rotated from $\mathrm{H} / / c$ to $\mathrm{H} / / a b$.

The angular dependence of the resistivity for the $\mathrm{Fe}_{1.04} \mathrm{Te}_{0.6} \mathrm{Se}_{0.4}$ at $14 \mathrm{~K}$ is shown in the inset of Fig. 5. All the curves have a symmetric cup-like shape, and the minimum value is at $\theta=90^{\circ}$. According to the anisotropic Ginzburg-Landau model, the effective upper critical field, $H_{\mathrm{c} 2}^{\mathrm{Gl}}(\theta)$, can be characterized as ${ }^{17}$

$$
H_{\mathrm{c} 2}^{\mathrm{GL}}(\theta)=H_{\mathrm{c} 2, \mathrm{ab}} /\left(\sin ^{2} \theta+\Gamma^{2} \cos ^{2} \theta\right)^{1 / 2},
$$

where $\Gamma$ is the anisotropy of the sample. As the resistivity in the mixed state depends on the effective field, ${ }^{30}$ the angular dependence of the resistivity can be scaled as $\rho=\rho_{\mathrm{o}} f(H) /$ $H_{\mathrm{c} 2}^{\mathrm{GL}}$, where $\rho_{\mathrm{o}}$ is the temperature independent part of the resistivity. Then, the resistivity measured under different magnetic fields should collapse into one curve at a certain temperature if the anisotropy parameter is properly scaled. Good scaling behaviour can be obtained for $\mathrm{Fe}_{1.04} \mathrm{Te}_{0.6} \mathrm{Se}_{0.4}$ with $\Gamma=2.5$ at $\mathrm{T}=14 \mathrm{~K}$, as shown in the main panel of Fig. 5. This value is higher than the obtained value of $\Gamma=2$ for $\mathrm{Fe}_{1.11} \mathrm{Te}_{0.6} \mathrm{Se}_{0.4}$ single crystal. ${ }^{31}$ It should also be mentioned that the anisotropy values were obtained using different methods, GL theory in our work, and the ratio of the upper critical field along the $a b$-plane and to that along the $c$-axis.

In summary, we have studied the pinning mechanism, pinning potential, and anisotropy of $\mathrm{Fe}_{1.04} \mathrm{Te}_{0.6} \mathrm{Se}_{0.4}$ single

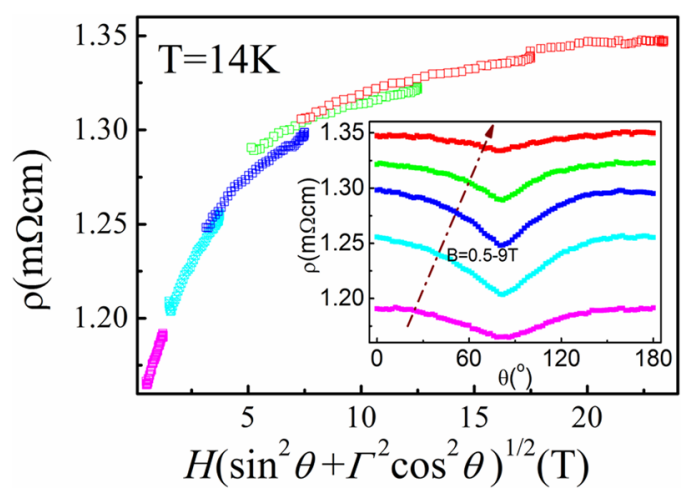

FIG. 5. Scaling of the resistance as a function of $H /\left(\sin ^{2} \theta+\Gamma^{2} \cos ^{2} \theta\right)^{1 / 2}$, based on GL theory at $\mathrm{T}=14 \mathrm{~K}$. Inset shows the angular dependence of the resistivity at $14 \mathrm{~K}$ under different magnetic fields. crystal. The obtained values of $p$ and $q$ based on the DewHughes model indicate the existence of $\delta l$ pinning, with a mixture of surface and point core pinning in this compound. In addition, the pinning potential value was obtained using the thermally activated flux flow model. The anisotropy value was obtained using GL theory.

This work was supported by the Australian Research Council (ARC) through Discovery Projects DP 1094073.

${ }^{1}$ Y. Kamihara, T. Watanabe, M. Hirano, and H. Hosono, J. Am. Chem. Soc. 130, 3296 (2008).

${ }^{2}$ X. H. Chen, T. Wu, G. Wu, R. H. Liu, H. Chen, and D. F. Fang, Nature 453, 761 (2008).

${ }^{3}$ G. F. Chen, Z. Li, D. Wu, G. Li, W. Z. Hu, J. Dong, P. Zheng, J. L. Luo, and N. L. Wang, Phys. Rev. Lett. 100, 247002 (2008).

${ }^{4}$ R. Zhi-An, Y. Jie, L. Wei, Y. Wei, S. Xiao-Li, L. Zheng-Cai, C. GuangCan, D. Xiao-Li, S. Li-Ling, Z. Fang, and Z. Zhong-Xian, EPL 82, 57002 (2008).

${ }^{5}$ W. Hai-Hu, M. Gang, F. Lei, Y. Huan, and Z. Xiyu, EPL 82, 17009 (2008).

${ }^{6}$ M. Rotter, M. Tegel, and D. Johrendt, Phys. Rev. Lett. 101, 107006 (2008).

${ }^{7}$ A. S. Sefat, R. Jin, M. A. McGuire, B. C. Sales, D. J. Singh, and D. Mandrus, Phys. Rev. Lett. 101, 117004 (2008).

${ }^{8}$ X. C. Wang, Q. Q. Liu, Y. X. Lv, W. B. Gao, L. X. Yang, R. C. Yu, F. Y. Li, and C. Q. Jin, Solid State Commun. 148, 538 (2008).

${ }^{9}$ X. Zhu, F. Han, G. Mu, P. Cheng, B. Shen, B. Zeng, and H.-H. Wen, Phys. Rev. B 79, 220512 (2009).

${ }^{10}$ H. Ogino, Y. Matsumura, Y. Katsura, K. Ushiyama, S. Horii, K. Kishio1, and. J.-i. Shimoyama, Supercond. Sci. Technol. 22, 075008 (2009).

${ }^{11}$ F.-C. Hsu, J.-Y. Luo, K.-W. Yeh, T.-K. Chen, T.-W. Huang, P. M. Wu, Y.-C. Lee, Y.-L. Huang, Y.-Y. Chu, D.-C. Yan, and M.-K. Wu, Proc. Natl. Acad. Sci. U.S.A. 105, 14262 (2008).

${ }^{12}$ A. Subedi, L. Zhang, D. J. Singh, and M. H. Du, Phys. Rev. B 78, 134514 (2008).

${ }^{13}$ B. C. Sales, A. S. Sefat, M. A. McGuire, R. Y. Jin, D. Mandrus, and Y. Mozharivskyj, Phys. Rev. B 79, 094521 (2009).

${ }^{14}$ K. W. Yeh, C. T. Ke, T. W. Huang, T. K. Chen, Y. L. Huang, P. M. Wu, and M. K. Wu, Cryst. Growth Des. 9, 4847 (2009).

${ }^{15}$ M. H. Fang, H. M. Pham, B. Qian, T. J. Liu, E. K. Vehstedt, Y. Liu, L. Spinu, and Z. Q. Mao, Phys. Rev. B 78, 224503 (2008).

${ }^{16}$ R. Khasanov, M. Bendele, A. Amato, P. Babkevich, A. T. Boothroyd, A. Cervellino, K. Conder, S. N. Gvasaliya, H. Keller, H. H. Klauss, H. Luetkens, V. Pomjakushin, E. Pomjakushina, and B. Roessli, Phys. Rev. B 80, 140511 (2009).

${ }^{17}$ G. Blatter, M. V. Feigel'man, V. B. Geshkenbein, A. I. Larkin, and V. M. Vinokur, Rev. Mod. Phys. 66, 1125 (1994).

${ }^{18}$ P. Das, A. D. Thakur, A. K. Yadav, C. V. Tomy, M. R. Lees, G. Balakrishnan, S. Ramakrishnan, and A. K. Grover, Phys. Rev. B 84, 214526 (2011).

${ }^{19}$ C. S. Yadav and P. L. Paulose, Solid State Commun. 151, 216 (2011).

${ }^{20}$ Y. Liu and C. T. Lin, J. Supercond. Novel Magn. 24, 183 (2011).

${ }^{21}$ C. P. Bean, Phys. Rev. Lett. 8, 250-253 (1962).

${ }^{22}$ C. S. Yadav and P. L. Paulose, New J. Phys. 11, 103046 (2009).

${ }^{23}$ D. Dew-Hughes, Philos. Mag. 30, 293 (1974).

${ }^{24}$ S. L. Prischepa, M. L. Della Rocca, L. Maritato, M. Salvato, R. Di Capua, M. G. Maglione, and R. Vaglio, Phys. Rev. B 67, 024512 (2003).

${ }^{25}$ Z.-S. Wang, H.-Q. Luo, C. Ren, and H.-H. Wen, Phys. Rev. B. 78, 140501 (2008).

${ }^{26}$ Z. Bukowski, S. Weyeneth, R. Puzniak, P. Moll, S. Katrych, N. D. Zhigadlo, J. Karpinski, H. Keller, and B. Batlogg, Phys. Rev. B 79, 104521 (2009).

${ }^{27}$ H.-S. Lee, M. Bartkowiak, J.-H. Park, J.-Y. Lee, J.-Y. Kim, N.-H. Sung, B. K. Cho, C.-U. Jung, J. S. Kim, and H.-J. Lee, Phys. Rev. B 80, 144512 (2009).

${ }^{28}$ Y. Jia, P. Cheng, L. Fang, H. Luo, H. Yang, C. Ren, L. Shan, C. Gu, and H.-H. Wen, Appl. Phys. Lett. 93, 032503 (2008).

${ }^{29}$ J. Jaroszynski, F. Hunte, L. Balicas, Y.-j. Jo, I. Raiccaronevicacute, A. Gurevich, D. C. Larbalestier, F. F. Balakirev, L. Fang, P. Cheng, Y. Jia, and H. H. Wen, Phys. Rev. B 78, 174523 (2008).

${ }^{30}$ G. Blatter, V. B. Geshkenbein, and A. I. Larkin, Phys. Rev. Lett. 68, 875 (1992).

${ }^{31}$ M. Fang, J. Yang, F. F. Balakirev, Y. Kohama, J. Singleton, B. Qian, Z. Q. Mao, H. Wang, and H. Q. Yuan, Phys. Rev. B 81, 020509 (2010). 